\title{
Filigrane
}

Écoutes psychothérapiques

\section{Formation et transmission : la passion des origines. Entrevue avec Jean Bossé}

\section{Réal Laperrière}

Volume 15, numéro 1, printemps 2006

URI : https://id.erudit.org/iderudit/013528ar

DOI : https://doi.org/10.7202/013528ar

Aller au sommaire du numéro

Éditeur(s)

Revue Santé mentale au Québec

ISSN

1192-1412 (imprimé)

1911-4656 (numérique)

Découvrir la revue

Citer ce document

Laperrière, R. (2006). Formation et transmission : la passion des origines.

Entrevue avec Jean Bossé. Filigrane, 15(1), 38-56.

https://doi.org/10.7202/013528ar d'utilisation que vous pouvez consulter en ligne.

https://apropos.erudit.org/fr/usagers/politique-dutilisation/ 


\section{Formation et transmission : la passion des origines. Entrevue avec Jean Bossé}

réal laperrière

R. L. : Docteur Bossé, je débuterai notre entretien par une question assez personnelle, si vous le voulez bien.

J. B. : Oui.

R. L.: Lorsqu'on lit vos textes, et je pense en particulier à certains que je considère importants: Propos sur la filiation, Devenir psychanalyste, Où vont les psychanalystes, ou encore le texte sur la supervision — ces textes ont été beaucoup lus et ont inspiré beaucoup de gens - , on y voit votre intérêt pour la formation des psychanalystes, pour les institutions psychanalytiques, la supervision; tous ces textes sont animés par une passion pour la transmission. Et surtout pour la question de l'origine. Alors, d'où cette passion origine-t-elle chez vous?

J. B. : Spontanément, je dirais que cette question renvoie à une autre question, celle de comment je suis devenu psychanalyste, praticien psychanalyste;j'emploie volontiers ce terme de praticien en psychanalyse. Alors comment suisje devenu psychanalyste ou praticien de la psychanalyse? Il serait probablement possible de trouver dans mon enfance les éléments explicatifs de cet intérêt. Mais je commencerai par l'adolescence.

Durant cette époque trouble — ne l'est-elle pas pour chacun de nous? - j'ai assisté à des conférences sur la psychanalyse données à mon collège par les $\mathrm{D}^{\text {rs }}$ Karl Stern et Victorin Voyer. C'est à ce moment que s'est formulé pour la première fois dans ma tête le désir de pratiquer ce métier; j'y ai reconnu une possibilité d'ouverture, une voie pour moi. Je me suis dit: «Voilà ce que je veux faire» et le projet de travailler avec $\mathrm{D}^{\mathrm{r}}$ Stern et $\mathrm{D}^{\mathrm{r}}$ Voyer s'est élaboré dans ma tête. Et, aussi, celui d'entrer en analyse dès que j'en aurais les moyens financiers. J'ai eu l'opportunité de travailler avec ces deux psychiatres au cours de ma formation médicale. L'été suivant ma troisième année d'études de médecine, j'ai pu me faire embaucher comme interne à l'Institut Albert-Prévost, où ils travaillaient tous les deux, et j'ai passé quatre mois avec eux. Ce fut une expérience très importante que le contact avec, non seulement la psychiatrie, mais avec l'approche dynamique, psychanalytique. Une deuxième expérience marquante s'est présentée aussi au cours de ma formation psychiatrique à l'Institut Albert-Prévost. Ce fut un moment significatif.

R. L. : C'était à Prévost aussi ?

J. B. : Oui. J'y ai passé deux ans. Nous avons été plongés, dès les premiers jours, dans la pratique avec des patients gravement atteints, sans préparation préalable 
autre que notre formation médicale, très pauvre sur le plan psychiatrique. En fait, des quatre résidents, j'étais le seul à avoir suivi un stage de quatre mois auprès de psychotiques. Après trois ou quatre mois de ce travail «sur le tas », accompagné de rencontres cliniques quotidiennes avec le patron - quand elles avaient lieu, parce que celui-ci n'était pas toujours disponible — , le directeur de l'enseignement nous a réunis pour connaître nos impressions à propos de ces rencontres si angoissantes. Imaginez, nous étions mis en contact avec des psychotiques dès le premier jour de notre résidence et nous devions nous débrouiller seuls. Comme je ne m'étais pas encore formulé une idée claire, j'oserais dire une esquisse, de mon expérience personnelle, j'ai laissé les autres exprimer leur opinion. Je ne me reconnaissais pas du tout dans ce qu'exprimaient mes collègues qui demeuraient, en fait, dans des zones superficielles. Je dirais maintenant qu'ils parlaient pour faire plaisir au directeur et pour être bien vus de lui. J'ai alors pris la parole le dernier et livré mes expériences le plus sincèrement possible. À savoir qu'entre le patient et moi, il n'y avait pas tellement de différences, que je pouvais me reconnaître dans ce qu'il était. Mon témoignage suscita un tollé de protestations venant non seulement de mes collègues, mais surtout — fait le plus surprenant du directeur de l'enseignement, pourtant reconnu pour son expérience avec les psychotiques. «Souvenez-vous mon cher monsieur, me dit-il, qu'il y a des différences et qu'il faut que vous vous y teniez.» Je suis sorti de cette rencontre complètement démoli, en me demandant ce que je faisais dans cette galère, au point même de remettre en question mon choix de carrière. Ce qui s'est passé là, je le comprendrai quelques années plus tard en analyse et en étudiant Freud dans sa rencontre avec Charcot, en découvrant comment il s'était reconnu lui-même dans les patients de son maître. Dans cette réunion avec mes collègues et le directeur, j'avais exposé la prise de conscience de ma folie. La réaction des autres avait été l'expression de leur résistance devant le miroir que je leur présentais.

Une troisième expérience, maintenant. Elle consiste dans ma première rencontre avec mon analyste. C'est un autre moment significatif.

R. L.: Oui?

J. B. : À ce moment-là, à Paris, nous faisions notre demande d'admission dans une des sociétés de psychanalyse existantes avant même de commencer une analyse. À la fin de ma première rencontre, intense, avec mon analyste où, pour la première fois, je m'étais senti accueilli, profondément écouté, je m'attendais à commencer le plus rapidement possible cette analyse que je désirais depuis fort longtemps. C'était comme la suite logique de ce qui venait de se passer entre nous. Mais telle ne fut pas d'abord la décision de mon analyste. Au contraire, il m'annonça: «Je vous prendrai lorsque la Société aura statué sur votre candidature. » Je bondis ! Je protestai que s'il avait de la disponibilité, je voulais commencer dès maintenant, que la décision de la Commission de candidatures ne nous concernait pas pour le moment, que je verrais plus tard. Sa réponse vint après un moment de silence, pendant lequel je remarquai son malaise à la rougeur de son visage. Elle fut géniale. Il se leva et lentement alla à sa table de travail, prit son agenda et me 
trouva quatre heures de disponibilité. Et j'ai commencé mon analyse dès le lendemain.

Le plus énigmatique de cette expérience fut ma propre réaction de protestation. D'où venait-elle ? C'est à partir de cette question que s'engagea mon interrogation sur la formation, sur la transmission et sur le processus analytique qui doit être protégé de l'ingérence institutionnelle. Celle-ci peut venir de plusieurs sources : de l'institution et son mode de fonctionnement, de l'analyste et de son degré d'autonomie par rapport à l'institution, de sa propre formation, i.e. comment lui-même est devenu analyste, etc.

R. L. : Oui.

J. B.: L'analyste peut répéter et transmettre les erreurs de son évolution, qui deviennent alors des points d'ancrage à une identification fausse. Ce fut pour moi un moment important que celui où mon analyste, qui se disait membre de la Société, dut se démarquer de celle-ci et redevenir analyste pour répondre lui-même à ma demande d'analyse.

R. L. : Pour répondre à votre demande d'analyse.

J. B. : Oui. Voilà ce qui est important. J'ai alors compris combien il était essentiel que l'analyste garde sa neutralité et se démarque du projet de formation de son analysé. Cette neutralité est continuellement attaquée par sa position dans la Société, par sa présence d'analyste formateur et de didacticien auprès du candidat et par sa position de complicité dans l'analyse et dans le projet de devenir analyste. Plus subrepticement encore, par sa propre formation d'analyste et par sa capacité — ou incapacité — d'autonomie par rapport à l'institution. J'ai déjà soumis cette question à un membre respecté et engagé, à un moment donné, dans une fonction de pouvoir au sein de la Société. Alors que je le questionnais sur l'influence que cette responsabilité pouvait avoir sur l'analyse de candidats, il me répondit : «Il faut bien quelqu'un pour faire ce travail de gestion ; mais le patient ne le sait pas. » Voilà deux rationalisations, parce que le candidat sait très bien, à partir d'un moment donné, quel rôle joue son analyste dans la Société.

R. L. : Bien sûr.

J. B. : À partir du moment où les analysés se rencontrent, tout se sait. Ce qui soulève d'importants problèmes de confidentialité dans ces analyses. Je pense à cet autre important événement que constitua ma rencontre avec Francis Pasche, cet analyste qui s'avéra un maître important et sur lequel je me suis appuyé à mes débuts à Paris. Quand je suis arrivé là-bas, j'avais un poste d'externe - ce qui équivaut ici à un poste de résident - à l'hôpital Ste-Anne, dans le cadre d'une entente France-Québec d'échange de jeunes médecins. J'avais pour projet de travailler à la clinique externe de cet analyste que je connaissais par ses écrits et aussi par d'autres Canadiens qui avaient passés dans sa clinique.

R. L.: Vous n'étiez pas le premier.

J. B.: Non, je n'étais pas le premier. Plusieurs ont passé dans sa clinique. René Major, Philippe Moreau, Pierre Labbé, et quelques autres. Pasche prenait un nouvel externe à tous les ans. Après moi, il y eut Paulette Letarte. C'était un 
homme qui possédait une grande qualité d'accueil. Je lui ai exposé mon intérêt pour la psychanalyse et il m'a rapidement offert son appui pour que je puisse travailler avec lui. Il venait à la clinique deux ou trois fois par semaine. Je le voyais très fréquemment. Il m'écoutait, me regardait travailler, me confiait des patients extrêmement difficiles pour éprouver mes capacités. Je compris vite que ce n'était pas la psychiatrie qui m'intéressait, mais la pratique de la psychanalyse. Après trois, quatre mois, un matin, il arrive à la clinique. Ordinairement, c'est moi qui allais le saluer dans son bureau. Mais cette fois, il se précipite dans le mien. Il s'assied dans la chaise ordinairement réservée aux patients. Je le sentais fébrile et, quant à moi, je vivais un sentiment d'irréalité. Un homme que j'admirais pour sa grande culture, pour sa position dans la Société — il en était le président — se met à parler de lui-même, de sa pratique, de l'ambiguïté dans laquelle il se sent dans les entrevues avec ses analysés. Il ne pouvait plus tolérer les difficultés que sa fonction de président engendraient chez ses analysés - qui étaient probablement des candidats - dans le déroulement de leur analyse. Il me confia: «Vous savez, pour faire ce métier on doit rester un homme tout à fait ordinaire. Et j’ai bien dit ordinaire.» Il termina son mandat quelques mois plus tard avec un réel soulagement. Cette expérience m'a beaucoup marqué.

À cette époque, il y avait un mouvement de crise à l'intérieur de la Société de Paris. Elle venait de l'influence des autres groupes, de même que des conceptions critiques de Lacan sur la formation. Ces critiques le rejoindront plus tard dans son propre groupe, en 1967-68, où se produira une autre scission, qui provoqua la formation d'un quatrième groupe (Groupe IV). L'enjeu de cette scission fut, comme pour toutes les scissions antérieures d'ailleurs, les questions de la fonction des analystes et les différences de conceptions concernant la formation et la transmission. À l'intérieur de la Société de Paris se déroulait également le travail de réflexion sur la formation de Robert Barande. Et surtout celui de Conrad Stein — je veux en parler, parce qu'il fut très important pour moi — concernant le rôle négatif que l'entrée en formation joue sur le processus de la cure personnelle des candidats.

À propos de cette problématique, Stein à élaboré le concept de secteur réservé, ou protégé, du transfert. La prise en compte de ce fait dans l'analyse des candidats va l'amener, en 1967, à une remise en question de son rôle à la commission de l'enseignement. Il prenait conscience de la grande complicité des didacticiens dans le projet de devenir analystes des candidats et de l'influence néfaste de cette dernière dans la création de cet espace protégé, du fait de leur position à la Société et surtout à l'Institut. Didacticien ou non, l'analyste, selon Stein, doit se démarquer du projet de devenir analyste de l'analysé dès qu'il se présente dans la cure. J'ai été pour le moins étonné de cette réflexion et $\mathrm{j}$ 'ai repensé à ma propre formation. J'ai été aussi influencé par la scission du groupe lacanien et la formation du quatrième groupe avec Piera Aulagnier, François Perrier, Nathalie Perrier, Claude Chabreul, Jean-Paul Valabrega. Tous leurs débats ont fait l'objet de textes incontournables sur la formation, sur la didactique et sur les rapports entre la 
psychanalyse et ses institutions. Ces textes ont tous été publiés dans les premiers numéros de Topique.

R. L.: Vous avez donc été assez rapidement, je dirais même dès votre première rencontre avec votre analyste, mis en contact avec l'espèce de paradoxe des institutions psychanalytiques.

J. B. : Oui, absolument.

R. L. : Vous avez toujours été très critique de l'institution psychanalytique et ce, dès le départ.

J. B. : Oui.

R. L.: Et en même temps, vous n'avez pas voulu être un psychanalyste sans institution, c'est à dire un psychanalyste en dehors ou, comme on dit ici, dans la marge...

J. B. : Non.

R. L.: Vous avez toujours été très présent à la Société Psychanalytique de Montréal; dès ses débuts, vous avez participé à en faire une institution la plus compatible possible avec le processus analytique. Mais, en même temps, toujours avec cette réserve.

J. B. : Oui.

R. L.: Comme s'il y avait là un paradoxe qui ne s'était jamais vraiment résolu. Comment être à la fois dans l'institution et à la fois garder cette position d'analyste.

J. B. : Oui, garder son autonomie.

R. L. : Oui.

J. B. : D'ailleurs, quand vous m'avez posé la question sur pourquoi je vous avais dit «Préparez vous à en sortir.», quand vous m'avez demandé une lettre de référence...

R. L. : Oui. C'est une remarque que je n'ai jamais oubliée, parce qu'elle était assez déconcertante pour un individu voulant devenir candidat à la formation. Vous avez d'abord accepté d'écrire cette lettre de recommandation pour m'aider à y entrer, ce dont je vous suis reconnaissant, puis vous m'avez dit : «Maintenant, il va falloir que vous pensiez à en sortir ».

J. B. : Oui.

R. L. : Un peu comme si la question de l'autonomie, et peut-être celle de la capacité à être seul au sens de Winnicott — dont vous avez déjà traité dans un texte — doit être présente dès le début.

J. B. : Oui. C'est absolument vrai. C'est important de garder envers l'institution un esprit critique et une autonomie de pratique, parce que l'exercice de ce travail s'effectue seul dans son bureau. Il est donc important d'avoir sa propre théorisation en marche. Non pas qu'elle n'est pas liée à celle de Freud; elle l'est toujours. Elle doit être toujours en discussion avec celle de Freud. On pourra y revenir. Je parlais de tous ces débats qui ont eu lieu dans le quatrième groupe. Je les ai suivis, étudiés et je m'en suis servi pour éclairer ma propre pensée. Tout cela dit, voyez comme c'est lié à ma trajectoire personnelle. Car viendra ensuite la 
fondation de la SPM (Société psychanalytique de Montréal) à laquelle j'ai participé.

R. L.: Vous y étiez dès la fondation?

J. B. : Dès la fondation, j'étais même le secrétaire des réunions qu'ils appellent les réunions constituantes de la société. Oui.

R. L.: Donc vous avez été à l'origine?

J. B.: Oui. Étant donné que c'est moi qui rédigeais les procès-verbaux, j'étais à même de constater ce qui se passait dans ce groupe en voie de formation. Dès le point de départ, deux conceptions complètement opposées de la formation se sont affrontées. Une parlait d'une rencontre intime, qui est une analyse personnelle, dans et à partir de laquelle tout doit se construire. C'est d'abord par elle que se fait la transmission. D'ailleurs, vous savez que le terme allemand utilisé pour désigner le transfert, Übertragung, est aussi employé pour nommer la transmission. C'est le même mot.

R. L.: C'est le même mot?

J. B.: Le même, oui.

R. L. : Donc la transmission origine du transfert.

J. B.: Oui, principalement.

R. L. : Mais vous parliez de deux conceptions...

J. B.: La deuxième est une conception selon laquelle l'analyste a un schéma d'évolution déterminé. Il sait ce qu'est une analyse. Tout est prévu à l'avance. Même la théorie est déjà toute là, et on a seulement qu'à la suivre dans les textes de Freud. Il a tout dit et on a seulement qu'à appliquer ses théories. Le savoir est établi, constitué, et le modèle, prédéterminé. Et ce qui n'est pas conforme au modèle, on le retranche. Pour paraphraser la phrase Freud, «Là où était le ça, le moi doit advenir», ici on trouvera l'idéal du moi et le surmoi de l'analyste ou de la Société ou de l'Institut. Et ainsi le savoir sera transformé en pouvoir. En d'autres mots, le pouvoir sera érigé en savoir privilégié sur l'inconscient. Il nous fallait donc sortir la psychanalyse du monde médical et de la psychiatrie. Elle était jusque-là considérée comme une spécialisation de la psychiatrie. L'organisation de la Société canadienne de psychanalyse était inspirée de celle de l'université et de ses facultés. Certains parlaient même, à cette époque, d'une faculté de psychanalyse. J'ai alors proposé de former un atelier continu sur l'enseignement. Nous y avons discuté tous les problèmes de la formation, de l'enseignement, de la transmission. Le secrétaire d'alors, qui avait organisé avec moi cet atelier, faisait le pont entre celui-là et le Conseil. Ainsi, ce dernier fut amené à réfléchir aux problèmes de la transmission et à accepter certains changements importants: ouverture de la formation aux non-médecins, participation des analystes à l'enseignement, changement dans l'habilitation des nouveaux analystes. Le travail de cet atelier a donc été très fécond. Je pense qu'il a servi de pont indispensable et qu'il permettait, en même temps, au Conseil d'alimenter sa réflexion. Mais c'est dans l'après-coup que je dis cela, car je crois que cet atelier a eu un impact. 
Les premiers colloques de la SPM $(1970,1971)$ ont porté sur la formation, la transmission, l'organisation de l'enseignement et de la Société. En 1979, la SPM proposa un autre colloque sur l'enseignement et j'y présentai un rapport sur la question. En 1985-1986, le Conseil eut l'idée d'organiser un panel sur la formation. Je proposai plutôt un atelier continu d'une durée d'un an. J'ai donc consacré beaucoup de temps à cette problématique. Après 1985, j'ai conclu que nous faisions fausse route. Il nous fallait plutôt renvoyer les analystes à l'analyse et les sensibiliser au problème créé dans la cure par la formation. C'est à dire, faire en sorte que les analystes des candidats soient éveillés à l'importance de se démarquer du projet de formation de ces derniers et de redevenir des analystes. Parce que ce qu'on voit souvent, ici surtout - en France c'est courant qu'un analyste se démarque du projet de devenir analyste de son analysé. Ici, non; le plus souvent, ici, il l'appuie.

R. L. : Ah oui?

J. B. : L'analyste l'appuie indirectement, en étant sur la liste des didacticiens. Et aussi souvent par ses propos et son attitude envers ce projet. Il n'a pourtant pas à le défendre, ni à le permettre, il doit l'analyser; point. C'est autre chose. Il doit sortir de sa fonction d'engendrement et de didacticien.

R. L. : D'engendrement?

J. B. : Oui.

R. L. : C'est à dire... le didacticien serait là pour former d'autres analystes ?

J. B. : Oui, exactement. Quand il adopte cette attitude, il ne fait plus d'analyse. Il sort de son rôle qui consiste à trouver, dans le transfert, le sens du projet de devenir analyste de son analysé. Ce projet apparaît d'abord comme un agir qui exige une analyse fine, rigoureuse, continue.

R. L.: Oui, c'est intéressant de penser comme un point aveugle le moment où un analysant élabore son projet de devenir analyste.

J. B. : Oui, justement.

R. L. : Point aveugle qui surgit parfois en cours de route dans une analyse.

J. B. : Il survient plus souvent qu'on ne le croit, parce que ce désir de devenir analyste comme son analyste fait partie du développement car, pour tout enfant, grandir se dit «faire comme». J'ai entendu des enfants me dire : «Mais comment on devient ce que tu fais? Moi, je voudrais devenir comme toi. » Derrière ce désir s'exprime celui de déloger son analyste et de le remplacer... C'est tout l'CEdipe qui se joue là, au fond.

R. L. : Évidemment avec la culpabilité et l'angoisse que ça peut générer.

J. B. : Bien sûr.

R. L. : J'aimerais revenir encore à la question de l'origine parce que vous avez travaillé sur ce que Octave Mannoni a appelé l'analyse originelle.

J. B. : Oui.

R. L.: On a longtemps parlé de l'auto-analyse de Freud jusqu'à ce que des auteurs comme Mannoni...

J. B. : Oui, en 1975. 
R. L. :... donc tard dans le développement de la psychanalyse, des auteurs comme Mannoni nous ont éclairés sur le fait qu'il y avait eu une forme d'analyse chez Freud.

J. B. : Bien oui, une analyse.

R. L. : Avec tout ce qu'il en est de la maladie transférentielle dans son rapport...

J. B. : À Fliess. Avec les symptômes, d'ailleurs; vous vous souvenez: «des troubles cardiaques », «des problèmes de cœur ».

R. L. : Et, vous avez approfondi cela...

J. B. : Oui, absolument.

R. L. :... dans un texte que vous aviez présenté d'ailleurs...

J. B. : Dans un colloque de la Société.

R. L.: Vous avez étudié la correspondance entre Freud et Fliess.

J. B. : Oui.

R. L.: Il doit bien rester quelque chose aujourd'hui, chez les analystes, de cette analyse originelle.

J. B.: Je ne le sais pas. Je parcourais, par exemple, des articles qui ont paru dans le dernier Bulletin (Bulletin de la Société psychanalytique de Montréal). Je suis frappé par le fait que très peu d'auteurs se réfèrent à l'analyse de Freud ; ils parlent beaucoup d'étudier Freud. On se réfère à lui comme à une sorte de théorie à apprendre, même à posséder, mais on fait peu allusion à l'analyse personnelle, à ce qui se passe dans cette dernière vis-à-vis de la relation transférentielle qui s'y développe. Toute une théorisation se déclenche pourtant, à ce moment-là. Si l'analyse est bien conduite, l'analysé, comme Freud, développe une théorisation sur son propre transfert.

R. L.: Une théorisation de son expérience analytique?

J. B.: Oui. Que se passe-t-il entre moi et mon analyste ? Et tout ce qui est dit. L'analysé développe une conception de cette relation. Comme cela s'est passé chez Freud. Qu'est-ce qu'il a fait Freud? L'interprétation des rêves est au fond un témoin, je pourrais dire pas à pas, de sa relation analytique. C'est à Fliess qu'il a dédié son volume.

R. L.: Oui, tout à fait.

J. B.: Oui. Comme d'autres aussi, je dirais même. Quand Fliess lui a fait une interprétation un peu sauvage sur le fait qu'il pouvait projeter ses propres pensées dans ses dires, Freud s'est fâché : «Puis, qui jette à la poubelle ce livre que je viens de te dédier? Parce que tu es dedans. Il n'est question que de toi là-dedans. De toi et de moi.» Il l'a dit comme ça! Ce qui montre qu'il avait compris ce qui s'était passé. Il était en train de comprendre l'importance de cette relation affective qui avait été pour lui un début de recherche intense, qu'il maintiendra jusqu'à la fin de sa vie. Dans Analyse finie et infinie il fait allusion à cette relation.

R. L. : Mais ce que vous dites m'amène à un étonnement. On a ici un programme de formation, ce qu'on appelle le séminaire des textes freudiens, qui est actuellement remis en question...

J. B. : Oui, 
R. L. : À mon souvenir, quand je suis passé à travers ces années de textes freudiens, La naissance de la psychanalyse, c'est à dire la correspondance FreudFliess, ne faisait pas partie de ce qui était présenté aux candidats.

J. B. : Non. Les psychanalystes n'ont pas reconnu toute l'importance de cette correspondance dans l'origine de la psychanalyse. Les lettres sont à mon avis fondamentales pour l'ensemble des analystes. Je pense que c'est un coup de génie de la part de Marie Bonaparte et de Ernst Kris d'avoir compris leur importance et de les avoir publiées. J'ai répertorié les sorties de La naissance de la psychanalyse à la bibliothèque de la Société et, du côté francophone, je pense que de 1980 à 1998 il n'a été sorti qu'une fois ou deux. Puis du côté anglophone, l'édition, complète, n'a jamais été sortie de la bibliothèque.

R. L. : Est-ce parce que tous les analystes l'ont acheté et l'ont à la maison ?

J. B. : J'en doute. Et je pense que très peu de gens ont lu L'analyse originelle d'Octave Mannoni et le travail d'Anzieu sur l'auto-analyse de Freud où l'auteur finit par reconnaître qu'elle se situait au niveau du transfert. Didier Anzieu a beaucoup parlé d'auto-analyse ; on lui a rétorqué qu'il n'y avait pas d'auto-analyse sans analyse personnelle. D'ailleurs, Laplanche l'affirme clairement, l'autoanalyse s'adresse toujours à quelqu'un. Et on pris beaucoup de temps à l'admettre. Cette lenteur est due à une idéalisation de Freud de laquelle les analystes ont pris bien du temps à sortir. Les travaux de Patrick Mahony sur Freud ont d'ailleurs contribué à cette désidéalisation; il a tenté de ramener ce dernier à une dimension beaucoup plus humaine, égalitaire. Mais, à mon avis, dans la correspondance Freud-Fliess se trouve représentée une théorie de la transmission, un chemin par lequel chaque analyste doit passer. C'est-à-dire, une analyse personnelle, la découverte de son fonctionnement psychique, de l'inconscient, du transfert. C'est en cherchant à comprendre sa relation avec son analyste que l'analysé jette les bases d'une première compréhension de sa psyché.

R. L.: Mais j'en reviens aux textes de Freud, parce qu'on se trouve dans une époque où plusieurs sociétés de psychanalyse, en fait plusieurs instituts, remettent en question l'importance qu'un candidat doit accorder à la lecture de Freud durant ses années de formation. Est-ce qu'on devrait passer tout ce temps sur Freud? On a entendu l'argument: "Après tout on ne lit plus Newton en physique, aujourd'hui. Alors pourquoi lirait-on encore Freud? Puisqu'on a maintenant des auteurs tels que Green, Kernberg, Aulagnier, Roussillon, pourquoi se taper encore Dora?» J. B. : Bon, je pense qu'on n'a pas assez saisi ce que Laplanche nous dit des textes de Freud. Il a pourtant passé sa vie à les étudier... Il y a, bien sûr, dans les textes de Freud une théorie, mais il y a aussi une méthode, la méthode analytique. Et la lecture de Freud doit tenir compte de cette méthode. D'ailleurs, j'ai suivi les cours que Laplanche a donné à l'École normale supérieure. Il développait, cette annéelà en 1965-1966, la relation d'objet et nous introduisait à une lecture de Freud qui tenait compte de la méthode analytique.

R. L. : Donc, la lecture de Freud sous l'angle de la méthode fait en sorte de désidéaliser la théorie pour davantage s'intéresser au processus? 
J. B. : Oui.

R. L. : C'est à dire à la façon dont il travaille les choses.

J. B. : Oui, bien sûr. L'Interprétation des rêves est le meilleur livre à cette fin. C'est vraiment un livre extraordinaire, en tout cas pour moi, et qui illustre la méthode du commencement à la fin; excepté peut-être le premier chapitre, assez ennuyeux parce qu'il répertorie les écrits sur le sujet. Mais à partir du moment où Freud assoit sa propre démarche, le livre devient extrêmement intéressant. Un grand livre qu'on ne se lasse pas de lire, de relire et de travailler. D'ailleurs, je suis encore en train de le travailler.

R. L.: Ah oui ?

J. B. : Oui. Cette publication s'est avérée un moment très important de l'histoire de la psychanalyse. Même le médecin de Freud, Marc Schur, devenu psychanalyste à New York après la mort de son patient, n'a pas vu ce niveau de transfert. Il parle de l'auto-analyse de Freud comme s'il l'avait faite seul. Pourtant, Schur élabore beaucoup sur l'effet néfaste de la rupture entre Freud et Fliess. Freud a toujours tenté d'aller au-delà de cette relation ; il était complètement piégé avec ses propres élèves à cause de l'échec de son lien à Fliess. Il y a tous ces gens qui ont été piégés par cette rupture. Ce n'est pas parce qu'on quitte son analyste que le transfert est terminé. Au contraire, jusqu'à ce que l'analysé puisse faire retour làdessus, le transfert demeure enkysté et continue de créer des difficultés.

R. L.: Et qu'en est-il quand on retrouve son analyste comme collègue à la Société de psychanalyse?

J. B. : Je pense que les relations avec l'analyste en resteront toujours un peu marquées. L'analyste se maintiendra toujours, en tout cas pour moi, à une certaine distance. Cela ne veut pas dire qu'il ne peut échanger avec ses anciens analysés. Mais il demeurera toujours qu'il a été leur analyste. Ce fait marque le contact, je crois.

R. L. : Vous avez dû connaître cette expérience, avec les années, de vous retrouver avec de vos analysants devenus membres de votre société.

J. B. : Oui.

R. L.: Devenus collègues.

J. B. : Oui. Ça provoque une relation assez contenue. Je crois que ce passage reste.

R. L. : Je vous amènerais maintenant sur un autre terrain. On a parlé de l'institution psychanalytique. Mais je ne pourrais pas m'entretenir avec vous sans qu'on parle un peu de votre travail dans l'institution psychiatrique où vous avez été longtemps...

J. B.: Oui.

R. L. : Vous avez tenu pendant combien d'années déjà ce séminaire sur la psychanalyse d'enfants, qui avait lieu en institution psychiatrique?

J. B.: Trente ans. Il a été fondé en 1969.

R. L. : C'est ça. Au moins trente ans. En trente ans, beaucoup de personnes sont passées là...

J. B. : Oui. Et même plusieurs de la Société. 
R. L. : Plusieurs et je vous avouerai, pour moi-même, que mes sept ou huit années de présence à ce séminaire ont grandement contribué à ma décision d'abord de retourner en analyse, puis de faire la formation à l'Institut. Ce séminaire a été important pour beaucoup de gens. Il portait sur la psychanalyse d'enfants et deux questions me viennent. La première est en rapport avec les psychanalystes en institution psychiatrique, comme l'institut Albert-Prévost, où se trouvait une clinique de pédopsychiatrie. J'aurais envie de vous demander qu'est-ce que les psychanalystes font là? Et l'autre question concerne la psychanalyse d'enfants elle-même. Mais celle-la, on pourra y revenir après.

Pour vous qui avez travaillé si longtemps dans une institution psychiatrique, qu'est-ce qu'un psychanalyste peut faire dans une clinique de pédo-psychiatrie ?

J. B. : Vous savez, les psychanalystes se sont avérés un problème à Prévost. En ce sens que, du côté de la clinique adulte, en tout cas, ils se sont plutôt cantonnés dans un rôle d'enseignants, de superviseurs, bien que quelques-uns aient fait un peu de pratique. Mais en infantile, moi, j'ai en ai toujours fait. Je trouvais important dans mon rôle d'enseignant, de superviseur, de faire de la pratique au même titre, avec la même clientèle que ceux qui venaient me voir en supervision. On travaillait dans le même bassin de population. Et puis, je jugeais important qu'ils me voient travailler: recevoir les gens, présenter les cas que je venais de voir. Plusieurs séminaires ont été consacrés à ce genre de matériel, vous vous en souvenez?

R. L. : Oui.

J. B. : À la sortie d'une séance de psychothérapie avec un enfant ou un adolescent, j'éprouvais parfois le besoin d'en parler et le séminaire était là. Il me servait à élaborer quelque chose qui s'était présenté dans la séance. J'ai décidé, à un moment donné, vers les années 1970, de sortir de mon rôle de psychiatre — parce que j'étais d'abord un pédopsychiatre à Prévost. J'ai donc démissionné de mon poste de directeur de secteur. Pourtant, c'est moi qui avais créé le secteur de psychiatrie Prévost, selon la formule du XIII ${ }^{\mathrm{e}}$ arrondissement à Paris, qui était une formule de psychiatrie de secteur: on déterminait un secteur de population et on s'occupait des cas qui en venaient. Je travaillais avec les professionnels de ce secteur, je rencontrais les psychologues, les travailleurs sociaux, les professeurs ; une dynamique s'est ainsi créée chez les professionnels autour de ce secteur. C'était très intéressant. Je crois toujours que c'est une façon intelligente de travailler en psychiatrie au moindre coût, même si on me reprochait que l'équipe comprenait beaucoup trop de personnes: quelques douze personnes pour couvrir Laval, une population de deux cents mille habitants qui générait une clientèle de cinq mille enfants ayant besoin de consultation... Quoiqu'on disait, c'était vraiment trop pour une équipe.

R. L. : Oui.

J. B. : C'était épouvantable. Un moment donné, on est devenu coincés comme dans un entonnoir, avec de nombreuses demandes auxquelles on ne pouvait plus répondre. Les pathologies étaient trop profondes pour que l'on puisse assurer un 
suivi adéquat. J'ai vu, à ce moment-là que je n'avais pas les moyens de continuer d'opérer sans un centre de jour, un centre d'observation, une clinique bien organisée. Je n'avais qu'une clinique externe et devais me débrouiller avec les malades qui décompensaient. J'ai compris, après de multiples interventions auprès des autorités, que je n'aurais jamais les ressources adéquates promises. C'est à ce moment-là que j'ai décidé de démissionner et de ne faire que de la pratique.

R. L.: C'est à dire que vous aviez décidé de ne plus avoir de fonction administrative.

J. B.: Oui. J'ai décidé de ne faire que de l'enseignement et de la clinique.

R. L.: Voir des patients.

J. B.: Oui, seulement. Ne plus participer aux comités de dossiers, ni à tous les comités qui se multiplient à l'hôpital, qui prennent tout notre temps de patients et finissent par nous identifier à des feuilles de papier.

R. L.: Vous ne vouliez pas être un gestionnaire.

J. B.: Non. pas du tout. Alors, j'ai fait de la pratique de thérapie d'enfants et d'adolescents. À la clinique de l'enfance et de l'adolescence, j'ai eu toute le liberté de pratique nécessaire, l'accord de tous les directeurs qui se sont succédés à la clinique. Une chance extraordinaire! Par ailleurs, du côté adulte, j'ai été informé qu'un collègue analyste s'était fait imposer de pratiquer une autre approche psychothérapique...

R. L. : Ah oui !

J. B. :... alors que c'était inacceptable ! Mais les professionnels à l'hôpital, vous le savez, sont instrumentalisés.

R. L. : Oui.

J. B. : Instrumentalisés... C'est-à-dire qu'on leur dit quoi faire et dans quelle direction le faire, et si les professionnels refusent, on ne leur confie plus de patients. Alors ils restent à croupir dans leur coin; ils perdent leur temps à attendre les patients.

R. L.: Alors, vous avez eu la chance de pouvoir travailler à votre façon?

J. B. : Oui, absolument. On me laissait faire. Et si cela n'avait pas été possible, je serais parti. Par ailleurs, j'ai changé ma pratique hospitalière après quelques années.

R. L. : Comment?

J. B. : Je m'étais rendu compte que les enfants ne restaient en thérapie que pour une trop courte période de temps, nous n'allions pas jusqu'au bout de la démarche. Un tel phénomène est presque normal chez les adolescents; les adultes, eux, partent et reviennent; mais les enfants, ce ne sont pas eux mais leurs parents qui mettent fin au traitement. J'ai donc entrepris une recherche sur ce phénomène avec des collègues et j'en suis venu à adopter une autre attitude. J'ai allongé le temps de l'évaluation: je prenais un mois, deux mois, trois mois parfois, pour étudier la situation. Et je me suis aperçu d'un phénomène étonnant: il s'installait quelque chose, une réflexion; un espace se créait. Il était important que cet espace puisse se dégager, autant du côté des parents que de celui de l'enfant, pour qu'une 
expérience thérapeutique puisse s'engager. J'ai remarqué, à partir de ce momentlà, que, dans bien des cas, les enfants restaient un peu plus longtemps. J'ai suivi des adolescents durant trois ou quatre ans, i.e. de l'âge de douze, treize ans à celui de dix-sept, dix-huit ans — c'est assez rare de travailler avec eux jusqu'à ce qu'ils règlent complètement leurs problèmes. Ils devenaient à même de continuer seuls, et les enfants aussi. Je me suis appliqué dans ces cas-là à me tenir au plus près à la cure type. C'est à dire à appliquer la même méthode analytique que dans la cure d'adultes, avec des variables, bien sûr, parce qu'on utilise le dessin, le jeu, le jeu de rôle pour créer un dialogue avec les enfants. Donc, je me suis appliqué à fonctionner à l'hôpital comme à mon bureau privé. Ce qui m'amène à une autre expérience que j'ai aussi réalisée. Quand j'ai quitté la clinique, j'avais encore un certain nombre d'adolescents en traitement. Je leur ai offert de venir à mon bureau privé. Il y en a quatre ou cinq qui sont venus.

R. L. : Et ?

J. B. : Et ils m'ont tous dit la même chose : qu'à mon bureau privé, ce n'était pas la même chose. Étonnant, non ? J'en ai déduit que je n'étais pas le même en bureau privé, alors qu'à l'hôpital, j'avais pourtant fait un effort pour me garder dans la même disposition qu'en privé.

R. L. : Vous n'étiez pas le même à votre bureau ?

J. B.: L'ambiance n'était pas la même, parce qu'elle comprenait un autre type d'accueil, un autre type d'espace physique. Une salle d'attente leur était réservée, toute à eux. Il n'y avait pas d'intermédiaire, de secrétaire. Ils n'avaient affaire qu'à moi. Tout se passait entre nous. L'espace d'intimité était beaucoup plus grand et ils s'en rendaient compte. «C'est pas pareil venir ici. C'est beaucoup plus intime. C'est beaucoup... Je ne suis pas pareil et vous n'êtes pas pareil. » Sans dire plus que ça. Donc, ils avaient senti un changement. C'était intéressant.

R. L. : Donc, quelque chose de la fonction de l'institution...

J. B. :... intervient à notre insu, oui.

R. L. : Peut-être parfois au bénéfice même du traitement?

J. B.: Oui. Je me suis même demandé si une analyse, ou une thérapie, était vraiment possible à l'hôpital.

R. L. : Qu'en pensez-vous?

J. B.: Je dirais oui, théoriquement. Mais jusqu'où n'est-elle pas toujours handicapée ? Enfin... La question demeure ouverte.

R. L. : Tout à fait. Et on revient à ma question de départ: qu'est-ce que les psychanalystes font dans une institution? Est-ce qu'on peut y faire un véritable travail analytique?

J. B. : Plusieurs essaient, en tout cas. Moi j’y suis arrivé. Auparavant, je crois qu'il faut prendre du temps pour installer une psychothérapie, c'est-à-dire installer des rencontres régulières. Pendant cette période d'évaluation prolongée, beaucoup de travail s'accomplit. Il ne faut pas bousculer les choses dans ce domaine.

R. L.: Revenons à la psychanalyse d'enfants, à votre séminaire. Parce que pendant, les trente années où ce séminaire a eu lieu, il était inscrit à l'Institut... 
J. B. : Il était inscrit un moment donné pour les candidats qui étaient là.

R. L. : Oui. Plusieurs personnes que je pourrais nommer ont passé par votre séminaire, du temps où ils étaient candidats, et sont maintenant psychanalystes à la Société. Mais depuis que vous avez mis fin à ce séminaire, aucun séminaire de la SPM porte sur la psychanalyse d'enfant, et pourtant on sait qu'elle en offre beaucoup.

J. B. : Oui.

R. L. : On a un séminaire sur l'observation de nourrissons, mais ce n'est pas la même chose. Comment pouvez-vous expliquer que dans une Société d'analystes il n'y ait pas de séminaire sur la psychanalyse d'enfants?

J. B. : Oui. À Paris, il y avait beaucoup de séminaires sur la psychanalyse et la thérapie d'enfants. Mais toujours en dehors de la Société.

R. L. : Comment expliquer ça ?

J. B.: La formation se faisait dans des centres extérieurs, à La Salpetrière par exemple.

R. L.: Oui.

J. B. : J'ai travaillé à La Salpetrière, avec les $\mathrm{D}^{\text {rs }}$ Widlocher et Lang, de même qu'au Centre psychopédagogique Claude-Bernard avec André Berge. J'y avais une clientèle; j'ai pratiqué dans ce centre durant toutes les années que j'ai passées en France. J'étais engagé comme psychothérapeute.

R. L. : Mais qu'est-ce qui fait que...

J. B. : Ici ?

R. L.: Qu'on ait si peu d'intérêt apparent. Parce que plein d'analystes travaillent avec des enfants un peu partout.

J. B. : Oui.

R. L.: Mais à la Société même, on n'en parle jamais.

J. B. : Oui, c'est exact.

R. L.: Et il existe ici, en théorie, une formation pour devenir psychanalyste d'enfants.

J. B. : Oui.

R. L. : Mais qui est donnée du côté anglophone et...

J. B. : Anglophone, oui.

R. L. : Et qui est très, très peu connue...

J. B. : Oui.

R. L. :... et peut-être pas très active.

J. B.: Oui. Je ne sais pas si ces gens voient encore des enfants.

R. L. : Alors ?....

J. B. : Alors qu'en Angleterre, par exemple, il se donne des formations en psychanalyse d'enfants. Mais je ne suis pas très au courant des modalités ni de l'organisation de cette formation.

R. L. : Je me suis déjà demandé si pour plusieurs psychanalystes — secrètement, parce que je ne crois pas que les analystes tiendraient un tel discours ouvertement - la psychanalyse d'enfants n'était pas vue un peu comme de la psychanalyse appliquée. 
J. B.: Qu'est-ce que vous voulez dire par appliquée?

R. L.: Une extension de la psychanalyse, mais pas vraiment de la psychanalyse.

J. B. : Mais, à Paris, beaucoup d'analystes pensaient ainsi. Il y avait trois options : la psychosomatique, la psychose et l'infantile. Il y en avait qui choisissaient les enfants, comme Diatkine, qui a toujours pratiqué avec les enfants. Lebovici. aussi. Tandis que d'autres, comme De M'Uzan, Fain, David Marty s'intéressaient aux maladies psychosomatiques. Et $\mathrm{D}^{\mathrm{r}}$ Racamier et son groupe travaillaient avec les psychotiques. La plupart des analystes qui traitaient les enfants les voyaient une fois par semaine.

R. L.: Oui.

J. B. : Une fois par semaine et ce n'est qu'un peu plus tard, avec Florence Bégouin, par exemple, qu'on a commencé à voir des enfants à une plus grande fréquence. À Claude-Bernard, je pense que j'étais le seul à voir des enfants plus d'une fois/semaine. Et c'était un enfant que j'avais en supervision avec André Berge. C'était donc exceptionnel. Diatkine se limitait à une fois par semaine. Dolto aussi. R. L.: Ah oui ?

J. B.: Même les adolescents, elle les rencontrait une fois par semaine. Elle ne voyait pas la nécessité de les rencontrer plus souvent. Elle disait que l'impact était suffisant et qu'ils évoluaient assez rapidement. C'est un fait. Je ne sais pas pourquoi ici on ne voit pas les enfants et les adolescents plus souvent.

R. L.: Oui.

J. B.: À la Société, peu de présentations portent sur des psychanalyses d'enfants.

R. L.: Oui.

J. B.: Pierre Drapeau a présenté assez fréquemment des cas sur des analyses d'enfants, un cas d'analyse d'enfant psychotique à deux reprises. Monique Meloche aussi.

R. L.: Au début de l'entrevue, vous avez insisté sur les termes «psychanalyste praticien». Puis quand vous avez parlé de votre expérience en institution, vous avez insisté sur le fait que vous pratiquiez dans l'institution. Pourquoi vous avez insisté sur ces termes?

J. B.: Parce que je pense qu'on est psychanalyste à son bureau avec le patient. C'est le patient, par sa demande, qui nous fait psychanalyste. Par sa demande et sa recherche. En dehors du bureau, je dirais qu'on n'est pas psychanalyste. On est un praticien qui témoigne de sa pratique à la Société. Je fais partie d'une Société dont les membres font le même travail que moi, sont des praticiens d'analyse. Mais je me demande si l'emploi du terme psychanalyste en-dehors de son bureau n'est pas un peu prétentieux. Comme le dit très bien Pontalis, «la prétention d'un savoir spécial dans la société, sur n'importe quel problème». Je vois des gens, par exemple, parler d'homosexualité en signant «psychanalyste».

R. L.: Oui. On invite même des psychanalystes à donner des avis.

J. B. : Comme s'ils possédaient un savoir spécial. Moi, je ne le crois pas. En tous cas, j'ai mes réserves là-dessus.

R. L. : Donc, vous auriez des réserves sur la prise de parole hors séance des analystes par rapport à des problématiques comme la politique, le terrorisme... 
J. B. : Oui. c'est exact.

R. L. : Des sujets à propos desquels on interpelle les psychanalystes actuellement. J. B. : Oui. Il faut faire attention. Nous n'avons pas de savoir spécial. Je ne dis pas qu'il ne faut pas intervenir comme tout citoyen qui a des choses à dire. Mais que nous ayons un savoir spécial ? Je ne crois pas.

R. L.: Est-ce qu'un psychanalyste doit écrire?

J. B.: Oui. Ça c'est une problématique qui m'a beaucoup mobilisé.

R. L. : Notamment par votre implication dans le Bulletin de la Société.

J. B. : Oui, mais même avant ça. Il y a une vingtaine d'années, j'ai commencé à tenir un journal clinique. Je me suis appliqué à prendre des notes, par exemple, en sortant des séances pour les travailler. Et je me suis aperçu du bienfait d'une telle activité. Elle me permettait de développer ma pensée, de me dégager de mon transfert, de le travailler, de prendre conscience de jusqu' où tout ça allait. Je me rendu compte que cette prise de notes faisait partie du travail d'entre les séances et je me suis livré à cette écriture de plus en plus souvent. Elle est devenue une activité beaucoup plus importante, bien sûr, parce qu'il fallait toujours avoir des articles pour remplir le Bulletin, étant donné que les membres n'écrivent pas. Ils remettent constamment à plus tard, puis la date de tombée arrive et ils n'ont encore rien écrit, ce qui fait que le nombre de textes est insuffisant pour remplir le Bulletin. Alors, il faut que quelqu'un ait des textes dans son tiroir. C'est ce que moi et d'autres faisions; nous avions des textes en réserve. Puis cela devient une habitude. $\mathrm{Ce}$ journal clinique est devenu dans les dernières années une activité quotidienne où je reprenais les séances et les entre-séances; je me suis ainsi aperçu combien ces dernières étaient essentielles. Il y survenait des choses importantes, tout aussi capitales que celles qui se passaient durant la séance. Une réflexion s'installe, en effet, tant chez l'analyste que chez l'analysé au sujet de ce qui s'est passé dans l'entrevue, et elle s'établit en l'absence de l'autre. Il est intéressant d'observer ce que l'analysé retient de cet espace de réflexion. Nous arrivons tous deux à la séance suivante à une place différente de celle où nous nous sommes quittés. Il est important de suivre cette activité psychique. L'analysé n'en parle parfois pas. Il s'était pourtant passé une chose très importante à la séance précédente, mais l'analysé n'en reparle plus : «Oh oui, je n'y ai pas pensé.» On se demande alors comment s'est organisée cette résistance chez lui et comment il arrive à reprendre le fil de son analyse à propos de ce qui est arrivé. Il a besoin de se défendre, aussi... R. L.: Oui.

J. B. : Je me suis donc aperçu de l'importance de cet espace de l'entre-séance et pour l'un et pour l'autre. J'ai aussi effectué une expérience. J'ai demandé à des supervisés d'écrire sur les entre-séances avec leurs patients. «Écrivez donc ce qui s'est passé. » Certains s'installent dans l'écriture plus intensément que d'autres ; Je me suis aperçu qu'ils parvenaient beaucoup plus vite à une pensée personnelle.

R. L. : Ah oui.

J. B. : Oui. C'était une constatation émouvante. Quelqu'un se mettait à avoir des réflexions personnelles sur son travail intime. Mais ce n'est pas donné d'emblée à 
chacun de connaître cette expérience. Il y a beaucoup de résistances à écrire dans notre société; l'écriture n'y est pas coutumière; en France, elle l'est. Il est cependant important d'écrire tout le temps et cette activité est devenue pour moi une habitude; je possède des douzaines de cahiers de notes écrites sur toutes sortes de sujets. L'écriture devient une rencontre avec moi-même, c'est-à-dire le nonmoi, comme le dit Paul Valéry. Il a rempli une quantité de cahiers qu'on a découverts après sa mort. Je ne sais pas si c'est deux, trois ou quatre cents. On est en train de les publier. Il s'agit de rencontres très intimes qu'il faisait le matin; il se levait tôt et il écrivait «au plus près de l'aurore», selon son expression. Près de l'aurore, parce que près de la sortie du rêve. Il tentait de saisir un peu de ce qui s'est passé. Il s'agit de la rencontre avec son non-moi, c'est-à-dire l'étranger en soi : une façon de signifier une rencontre avec son inconscient. Cette réflexion commence dans le rêve et se poursuit à l'intérieur de soi. C'est quand même un médium extraordinaire pour rejoindre une connaissance de soi et la poursuivre. Non seulement de soi mais des autres aussi, c'est la même chose. C'est... Et surtout, à ce moment-ci de ma vie où j'ai décidé d'interrompre ma pratique. J'ai travaillé énormément cette question : comment faire cela?

R. L.: On va s'arrêter ici. Mais avant, je veux vous demander de me parler de votre décision d'interrompre, de la décision d'un analyste de prendre sa retraite.

J. B. : Oui.

R. L.: On dit souvent qu'un analyste ne prend pas sa retraite, n'arrête jamais et on entend beaucoup d'histoires d'analystes qui ont poursuivi, presque à la toute fin, leur travail avec des patients. Et c'est même valorisé, je dirais. Alors vous, vous avez pris la décision, il y a un certain temps, de ne plus prendre de patient en analyse...

J. B.: Oui, il y a deux, trois ans.

R. L. :... Alors, je vous le demande de ma position de plus jeune, parce que cela fait peut-être partie de ce que les aînés ont à nous transmettre, qu'est-ce qui fait qu'on arrive... Comment fait-on cela?

J. B.: Cela dépend de l'idée qu'on en a. J'avais toujours eu l'idée que je poursuivrais le plus longtemps possible. Mais en même temps j'avais la conviction que, pour pratiquer ce métier, il fallait être en bonne forme, tant physique que mentale, et être vigoureux, du fait qu'en tant qu'analyste, nous recevons quotidiennement des charges affectives très violentes et, qu'avec le temps, elles laissent des marques. Donc, un moment donné, notre opinion change; on se voit vieillir, devenir moins alerte, certaines infirmités ou handicaps font progressivement leur apparition. Alors... bon, j'en suis venu à la conclusion que la terminaison de la pratique de cette profession est un processus, une décision qui se prépare longtemps d'avance.

R. L. : Oui.

J. B.: Un processus doit s'installer et s'accomplir avant de prendre la décision. Une question m'a beaucoup aidé. Des gens s'occupent de vous, comme le médecin par exemple, et eux aussi vieillissent et s'arrêtent. Une femme, qui s'occupe de 
mes affaires depuis longtemps, m'a annoncé un jour qu'elle allait prendre sa retraite dans un an ou deux. Elle a ajouté: «Si j'avais à choisir quelqu'un pour s'occuper de mes affaires, est-ce que j'accepterais une personne comme moi ?» Cette confidence me servit de message. Je me suis dit: "Tiens, c'est la question que je dois me poser: Si j'avais à consulter un analyste, est-ce que je choisirais un analyste dans ma condition? Si je réponds oui, je peux continuer. Mais si je réponds non, ce serait mal avisé de poursuivre. Je pense que c'est une bonne façon de voir les choses ». Et c'est ainsi que j'ai pris la décision. Mais la retraite n'est pas un arrêt banal, quand a aimé passionnément la pratique de la psychanalyse, ce métier si riche en expériences humaines, en connaissance de nous-même et des autres. Il nous donne la possibilité de rencontrer des êtres tout à fait extraordinaires, il faut bien le dire, qui sont venus nous confier leur intimité. Les voir devenir maîtres de leur vie et pouvoir continuer leur chemin constitue un grand plaisir narcissique de bon aloi, si on peut dire... Il s'agit de la même expérience que de voir nos enfants grandir et se débrouiller dans la vie. Prendre sa retraite n'est pas une décision simple. Elle constitue un deuil très important qui doit s'effectuer lentement.

R. L. : Elle doit faire l'objet d'un travail.

J. B. : Ah oui, absolument. À tous les jours. Durant cette période, j'ai tenu un journal extrêmement important sur ce qui se passait. Comment pouvoir accepter, par exemple, l'agressivité des patients, car il arrive toujours que certains patients n'ont pas terminé, veulent revenir et continuer. J'ai même rencontré quelqu'un sur la rue, qui m'a reconnu et m'a téléphoné pour me dire: «Mais... c'est bien vous que j'ai rencontré sur la rue ?». Elle était une patiente assez difficile : certains psychotiques, en suivi, demeurent toujours là, près... Elle s'informait de ma présence. Je lui ai répondu: «Mais je vais devoir arrêter bientôt». La colère est sortie: «Vous n'avez pas le droit de faire ça!» Et pourtant, elle ne venait plus me voir depuis 10 ans et je ne l'avais vue que dix fois dans sa vie. C'est pour dire, ils nous gardent comme objet en réserve. Il faut donc être prêt à accepter cette agressivité, qui est très constructive d'ailleurs, car si elle n'est pas assez verbalisée, la continuité de l'analyse sera mise en jeu. Ces personnes ne pourront pas investir un autre analyste si cette colère n'est pas déposée chez nous. C'est très important.

R. L.: Tout à fait.

J. B. : Mon métier me manque cruellement à certains jours. Mais il y a plein de choses qu'on peut investir en dehors de l'analyse. On peut trouver un autre lieu de créativité, en fait. Il s'agit de chercher. Si on cherche, on trouve.

R. L. : Et vous avez trouvé?

J. B.: Oh oui, j'ai trouvé. Depuis une quinzaine d'années, je m'intéresse à la philosophie antique. J'ai étudié Socrate et sa maïeutique, qui ressemble beaucoup à la méthode psychanalytique. En étudiant Socrate, j'ai aussi scruté les dialogues de Platon que j'ai lus et relus : durant l'Antiquité, les philosophes étaient ceux qui traitaient les maladies de l'âme. Maintenant, je suis inscrit à des cours universitaires. 
R. L.: Oui.

J. B. : Ces grands philosophes étaient passablement avancés dans la connaissance de l'individu. Et même de la parole. Par exemple, Antiphon avait découvert que la parole était d'une importance extraordinaire. Il avait remarqué qu'en laissant parler les gens, ils allaient mieux. Alors il s'est mis à développer des boutiques de compassion pour aider les gens.

R. L. : De compassion?

J. B. : Parce qu'il devait gagner sa vie. Il n'était pas d'Athènes, alors que les Athéniens, eux, étaient nourris et logés par l'État. C'est intéressant de voir quelqu'un découvrir le pouvoir de la parole, comme Platon et Aristote l'ont aussi fait, d'ailleurs. Aristote disait que pour connaître l'origine d'une chose, il faut remonter à sa naissance. C'est bien ce que je fais, non?

R. L. : Oui. Je constate que cette question d'origine vous passionne toujours autant.

J. B. : Oui. Je suis dans l'origine de la pensée...

R. L. : Bien oui.

J. B. : ... la pensée humaine. Ces philosophes ont risqué leur vie pour essayer de mettre de l'ordre dans la pensée. Ils étaient beaucoup plus avancés que leurs concitoyens et ont ainsi risqué leur peau. Certains ont été éliminés. Socrate a été assassiné, Antiphon et Protagoras aussi. Aristote a été obligé de s'exiler parce qu'on lui préparait un procès pour impiété ou quelque chose du genre. Il était Macédonien et on sait comment la Macédoine, avec Alexandre, a dominé la Grèce durant un certain temps. Après la mort d'Alexandre, il a dû abandonner son école et ses élèves; pour un homme comme lui, c'était presque la mort. Il est d'ailleurs mort très peu de temps après. Il est mort à 60 ans, alors qu'à cette époque, les philosophes mouraient très vieux, soit à 70 ou 80 ans ; certains ont même vécu jusqu'à 100 ans.

R. L.: Alors, peut-être que dans quelques années on fera un entretien sur vos travaux philosophiques... On va s'arrêter maintenant.

Révision linguistique : hélène richard 